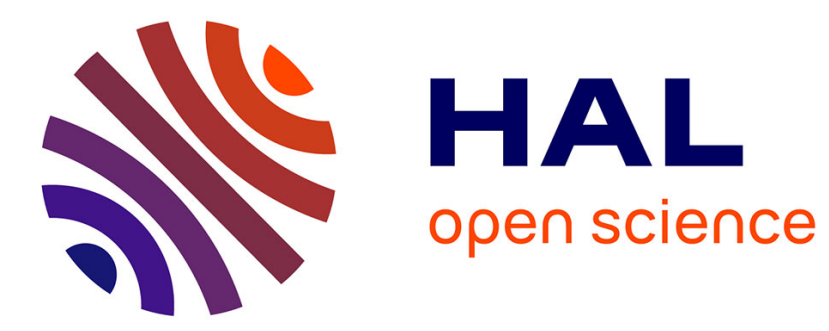

\title{
Uniform labelled calculi for conditional and counterfactual logics
}

Marianna Girlando, Sara Negri, Giorgio Sbardolini

\section{To cite this version:}

Marianna Girlando, Sara Negri, Giorgio Sbardolini. Uniform labelled calculi for conditional and counterfactual logics. WoLLIC 2019, Jul 2019, Utrecht, Netherlands. hal-02330313

\section{HAL Id: hal-02330313 \\ https://hal.science/hal-02330313}

Submitted on 23 Oct 2019

HAL is a multi-disciplinary open access archive for the deposit and dissemination of scientific research documents, whether they are published or not. The documents may come from teaching and research institutions in France or abroad, or from public or private research centers.
L'archive ouverte pluridisciplinaire HAL, est destinée au dépôt et à la diffusion de documents scientifiques de niveau recherche, publiés ou non, émanant des établissements d'enseignement et de recherche français ou étrangers, des laboratoires publics ou privés. 


\title{
Uniform labelled calculi for conditional and counterfactual logics *
}

\author{
Marianna Girlando ${ }^{1}$, Sara Negri ${ }^{2}$, and Giorgio Sbardolini ${ }^{3}$ \\ 1 Aix Marseille Univ, Université de Toulon, CNRS, LIS, Marseille, France and \\ University of Helsinki, Finland - marianna.girlando@univ-amu.fr \\ 2 University of Helsinki, Finland - sara.negri@helsinki.fr \\ 3 The Ohio State University - sbardolini.1@osu.edu
}

\begin{abstract}
Lewis's counterfactual logics are a class of conditional logics that are defined as extensions of classical propositional logic with a twoplace modal operator expressing conditionality. Labelled proof systems are proposed here that capture in a modular way Burgess's preferential conditional logic $\mathbb{P C L}$, Lewis's counterfactual logic $\mathbb{V}$, and their extensions. The calculi are based on preferential models, a uniform semantics for conditional logics introduced by Lewis. The calculi are analytic, and their completeness is proved by means of countermodel construction. Due to termination in root-first proof search, the calculi also provide a decision procedure for the logics.
\end{abstract}

Keywords: Conditional logics · Counterfactual logics · Proof theory · Preferential models · Labelled calculi

\section{Introduction}

In Stalnaker's and Lewis's approach, conditional logics are defined as extensions of classical propositional logic by means of a two-place modal operator, the conditional, here denoted as $>$. This intensional operator is intended to express a more fine-grained notion of conditionality than material implication.

Lewis introduced counterfactual conditional logics to extend formal reasoning to counterfactual sentences, i.e., statements of the form If Trump hadn't won the elections, Clinton would have been president. Other than counterfactual logics (system $\mathbb{V}$ and its extensions) conditional logics include a weaker family of systems: in this paper, we consider preferential conditional logic $\mathbb{P C L}$ and all its extensions. These latter systems have received attention in artificial intelligence since the conditional operator can be interpreted as expressing non monotonic inferences, i.e., sentences of the form Normally, cats are afraid of dogs. In particular, the fragment of $\mathbb{P} \mathbb{L}$ without nesting of the conditional operator is equivalent to system $\mathbf{P}$ of [14]. There are other applications of conditional logics in the fields of knowledge base update [13], causality [8] and, in an epistemic setting, belief revision $[4,12]$.

\footnotetext{
* This work was partially supported by the Academy of Finland research project no.
} 1308664 and by the project TICAMORE ANR-16-CE91-0002-01. 
The semantics of conditional logics is defined in terms of possible world structures in which, intuitively, a formula $A>B$ is true at world $x$ if $B$ is true in the set of worlds at which $A$ is true that are more similar, in a sense to be formalized, to $x$. Preferential models were proposed by Lewis and studied, among others, by Burgess, who proved completeness of $\mathbb{P C L}$ with respect to these models [5]. Halpern and Friedman extended the proof to extensions of $\mathbb{P C L}$ [7]. On a formal level, these models explicitly employ the notion of comparative similarity among worlds: they are defined by adding to a set of possible worlds $W$ a family of subsets $W_{x}$ for each $x \in W$, representing the worlds accessible from $x$, and a binary relation $\leqslant_{x}$, expressing similarity among worlds. Thus, $y \leqslant_{x} z$ means world $y$ is at least as similar as $z$ to world $x$.

In this article, we define a family of modular labelled calculi G3P* for conditional logic $\mathbb{P C L}$ and all its extensions, including counterfactual logics, i.e., $\mathbb{V}$ and its extensions. The calculi are based on preferential semantics: following the well-established methodology proposed by the second author, the calculi import into the sequent calculus the semantic elements of preferential models by means of syntactic elements (labels and relational symbols).

In [20], Negri and Sbardolini presented a labelled calculus based on ternary relations for a system of Lewis's conditional logic $\mathbb{V} \mathbb{C}(\mathbb{V}$ to which the condition of Centering is added). The present article stems form a comment in Weiss's thesis [22]: the author observes, correctly, that Negri and Sbardolini's proof system is actually adequate to capture the stronger system $\mathbb{V} \mathbb{C}(\mathbb{V} \mathbb{C}+$ Uniformity). This led us to an analysis of labelled calculi based on preferential models. It turns out that it is possible to define modular proof systems on the basis of these natural classes of models.

The article is organised as follows. In Section 2, conditional logics and preferential models are introduced. Section 3 presents the rules of the calculi $\mathbf{G 3 P}^{*}$ and Section 4 their structural properties. In Section 5, we define a proof search strategy that ensures termination in root-first proof search for the systems without the semantic conditions of Uniformity and Absoluteness. This allows to prove completeness of the calculi by extracting a countermodel from failed proof search. The conclusion (Section 6) gives a comparison of the calculi presented in this article with other proof systems for conditional logics found in the literature.

\section{Conditional logics and preferential models}

The language of conditional logics is defined by means of the following grammar, for $p$ propositional variable, $A, B \in \mathcal{L}_{\text {cond }}$, and $>$ the conditional operator:

$$
\mathcal{L}_{\text {cond }}=p|\perp| A \wedge B|A \vee B| A \rightarrow B \mid A>B
$$

An axiomatization of $\mathbb{P C L}$ is defined adding to the axioms and inference rules of classical propositional logic the following: 


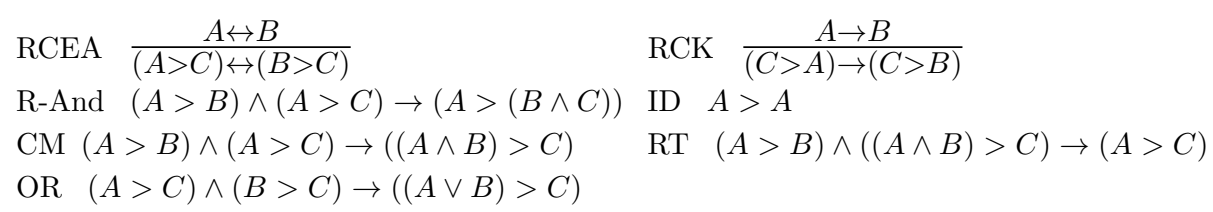

An axiomatization of Lewis's logic $\mathbb{V}$ is obtained by adding axiom $C V$ to $\mathbb{P C L}$. Extensions of both $\mathbb{P C L}$ and $\mathbb{V}$ are defined by adding the axioms for normality, total reflexivity, weak centering, centering, uniformity and absoluteness (denoted below by the corresponding initials). The family of systems is represented in Figure 1.

$$
\begin{aligned}
& \mathrm{CV}((A>C) \wedge \neg(A>\neg B)) \rightarrow((A \wedge B)>C) \\
& \mathrm{N} \neg(\top>\perp) \quad \mathrm{T} \quad A \rightarrow \neg(A>\perp) \\
& \mathrm{W} \quad(A>B) \rightarrow(A \rightarrow B) \quad \mathrm{C} \quad(A \wedge B) \rightarrow(A>B) \\
& \mathrm{U}_{1} \quad(\neg A>\perp) \rightarrow \neg(\neg A>\perp)>\perp \quad \mathrm{U}_{2} \quad \neg(A>\perp) \rightarrow((A>\perp)>\perp) \\
& \mathrm{A}_{1} \quad(A>B) \rightarrow(C>(A>B)) \quad \mathrm{A}_{2} \quad \neg(A>B) \rightarrow(C>\neg(A>B))
\end{aligned}
$$

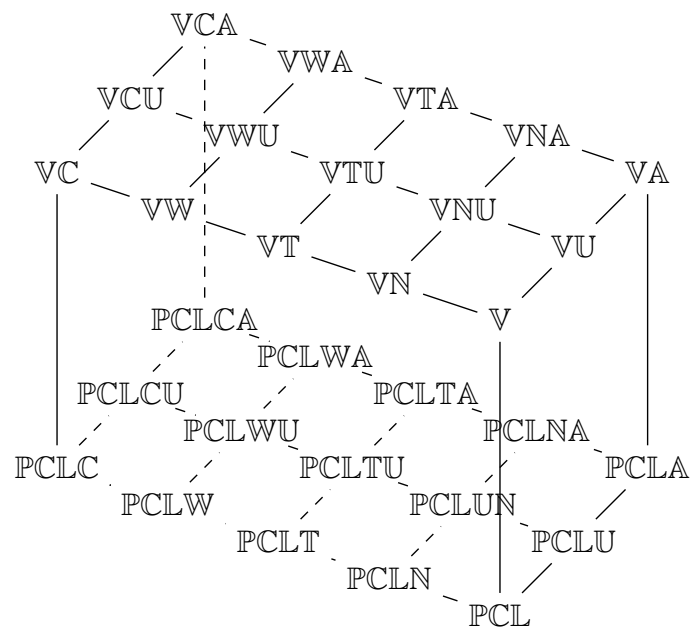

Fig. 1. The conditional logics cube

We use preferential models as the semantics for the conditional logics cube. Preferential models were studied, among others, by Burgess, who proved the adequacy of this class of models with respect to preferential logic [5].

Definition 1. A preferential model $\left\langle W,\left\{W_{x}\right\}_{x \in W},\left\{\leqslant_{x}\right\}_{x \in W}, \llbracket \rrbracket\right\rangle$ consists of a non-empty set of worlds, $W$, and for every world $x \in W$, a set $W_{x}$ of worlds accessible from it, and a binary relation $\leqslant_{x}$ over $W$. The propositional evaluation $\llbracket \rrbracket:$ Atm $\rightarrow \mathcal{P}(W)$ selects the worlds at which an atomic formula is true. The relation $\leqslant_{x}$ satisfies the properties of 
- Reflexivity, for all $w \in W, w \leqslant x$, and

- Transitivity, for all $w, y, z \in W$, if $w \leqslant_{x} y$ and $y \leqslant_{x} z$ then $w \leqslant_{x} z$.

The truth condition for the conditional operator within preferential models is:

$x \Vdash A>B \equiv$ for all $z \in W_{x}$, if $z \Vdash A$, then there exists $y \in W_{x}$ such that $y \leqslant x z, y \Vdash A$, and for all $k \in W_{x}$, if $k \leqslant_{x} y$ then $k \Vdash A \rightarrow B$.

Extensions of preferential models are specified by adding conditions on the relation $\leqslant_{x}$. These models are adequate for the logics in the conditional cube [7].

Definition 2. Extensions of preferential models are defined as follows:

- Normality: For all $x \in W, W_{x}$ is non-empty;

- Total reflexivity: For all $x \in W$ it holds that $x \in W_{x}$;

- Weak centering: For all $x \in W$, for all $y \in W_{x}$, it holds that $x \leqslant x y$;

- Centering: For all $x \in W$, for all $y \in W_{x}$, it holds that $x \leqslant x y$ and if there is $w \in W_{x}$ such that for all $y \in W_{x}, w \leqslant x$, then $w=x$;

- Uniformity: For all $x \in W$, for all $y \in W_{x}$ it holds that $W_{y}=W_{x}$;

- Absoluteness: Uniformity plus for all $w_{1}, w_{2} \in W_{x}, w_{1} \leqslant x w_{2}$ iff $w_{1} \leqslant y w_{2}$;

- Nesting: For all $x \in W$, for all $w_{1}, w_{2} \in W_{x}$, either $w_{1} \leqslant x w_{2}$ or $w_{2} \leqslant x w_{1}$.

Some of the above conditions are incremental: total reflexivity implies normality, weak centering implies total reflexivity, centering implies weak centering and absoluteness implies uniformity.

\section{$3 \quad$ Labelled proof systems}

In this section we shall define a family of modular calculi for the conditional cube. To this aim, we enrich our language with a sets of labels $x, y, z, \ldots$ denoting worlds in preferential models. Furthermore, we allow the following expressions to occur in sequents: labelled formulas $x: A$, denoting $x \Vdash A$, and relational atoms $y \in W_{x}, y \leqslant_{x} z$ and $x=y$, having the same meaning as their semantic counterparts. Following [20] we introduce an indexed modal operator, and reformulate the truth condition of $A>B$ in terms of this operator:

$$
w \Vdash \square_{x} A \equiv \text { for all } k \in W_{x} \text {, if } k \leqslant{ }_{x} w \text { then } k \Vdash A
$$

$(*) x \Vdash A>B \equiv$ for all $z \in W_{x}$, if $z \Vdash A$, then there exists $y \in W_{x}$ such that

$$
y \leqslant x z, y \Vdash A \text {, and } y \Vdash \square_{x}(A \rightarrow B) .
$$

Following [19], we introduce an indexed conditional operator to treat the second disjunct of the truth condition of the conditional operator:

$$
C_{x}^{z}(A, B) \equiv \text { there exists } y \in W_{x} \text { such that } y \leqslant{ }_{x} z, y \Vdash A \text { and } y \Vdash \square_{x}(A \rightarrow B)
$$

Thus, the truth condition for the conditional operator can be stated as follows:

$$
(* *) x \Vdash A>B \equiv \text { for all } z \in W_{x} \text {, if } z \Vdash A \text {, then } C_{x}^{z}(A, B) \text {. }
$$


Observe that the extension of the language is only at the level of the labelled rules and produces, in the course of proof search, only formulas of a certain specific form. Formulas containing the new operators never occur as proper subformulas of other formulas and an indexed modality can have only an implication in its scope.

The rules of the labelled proof systems are defined by analysing the truth conditions of the above operators (Figure 2). Rules Ref and Tr express reflexivity and transitivity of $\leqslant_{x}$; rule Ref $=$ and Repl express reflexivity of equality and the property of replacement of equals. We call G3P the calculus for $\mathbb{P C L}$; calculi for extensions are defined in a modular way by adding to $\mathbb{P C L}$ the rules corresponding to the semantic properties of $\leqslant_{x}$. We denote by G3P* the whole family of calculi. The condition of freshness of a variable $y$ in a rule is indicated by $(y !)$.

In order to prove soundness of the rules of the calculus we have to provide a definition of realization in a preferential model. The definition uses the operators of the extended language, and to guarantee its non-circularity we need to define a notion of weight of formulas:

Definition 3. Given a labelled formula $F$ of the form $x: A$, let the pure part of $F$ be defined as $p(x: A)=A$, and the labelled part as $l(x: A)=l\left(x: \square_{k} A\right)=x$. The weight of a labelled formula is an ordered pair $\langle\mathrm{w}(p(F)), \mathrm{w}(l(F))\rangle$ where

- for $x$ world label, $\mathrm{w}(x)=0$

$-\mathrm{w}(p)=\mathrm{w}(\perp)=1 ; \mathrm{w}(A \circ B)=\mathrm{w}(A)+\mathrm{w}(B)+1$, for $\circ$ conjunction, disjunction or implication; $\mathrm{w}\left(x: \square_{k} A\right)=\mathrm{w}(A)+1 ; \mathrm{w}\left(C_{x}^{z}(A, B)\right)=\mathrm{w}(A)+\mathrm{w}(B)+3$; $\mathrm{w}(A>B)=\mathrm{w}(A)+\mathrm{w}(B)+4$.

Definition 4 (Realization). Given a model $\mathcal{M}=\left\langle W,\left\{W_{x}\right\}_{x \in W},\left\{\leqslant_{x}\right\}_{x \in W}, \llbracket \rrbracket\right\rangle$, and a set $P$ of world labels, a P-realization over $\mathcal{M}$ is a function $\rho: P \rightarrow W$ that assigns to each world label $x \in P$ an element $\rho(x) \in W$. Satisfiability of a formula $F \in \mathcal{L}_{\text {cond }}$ is defined by cases as follows: $\mathcal{M} \vDash_{\rho} y \in W_{x}$ if $\rho(y) \in W_{\rho(x)}$; $\mathcal{M} \vDash_{\rho} y \leqslant_{x} z$ if $\rho(y) \leqslant_{\rho(x)} \rho(z) ; \mathcal{M} \vDash_{\rho} x: p$ if $\rho(x) \in \llbracket p \rrbracket$, for $p$ atomic; ${ }^{4}$ $\mathcal{M} \vDash_{\rho} w: \square_{x} A$ if for all $y \in W_{\rho(x)}$, if $y \leqslant \rho(x) \rho(w)$, then $y \Vdash A ; \mathcal{M} \vDash_{\rho} C_{x}^{z}(A, B)$ if there exists $y \in W_{\rho(x)}$ such that $y \leqslant_{x} \rho(z), y \vDash A$ and $y \vDash \square_{x}(A \rightarrow B)$; $\mathcal{M} \vDash_{\rho} x: A>B$ if for all $k \in W_{\rho(x)}$, if $k \vDash A$, then $k \vDash C_{x}^{k}(A, B)$. A sequent $\Gamma \Rightarrow \Delta$ is valid in $\mathcal{M}$ under the $\rho$ realization iff whenever $\mathcal{M} \vDash_{\rho} F$ for all $F \in \Gamma$, then $\mathcal{M} \vDash_{\rho} G$ for some $G \in \Delta$. A sequent is valid in a class of preferential models if it is valid under any realization for any model of that class.

The above definition immediately yields:

Theorem 1 (Soundness). If a sequent is derivable in $\mathbf{G 3} \mathbf{P}^{*}$, then it is valid in the corresponding class of preferential models.

Remark 1. The sequent calculi of Figure 2 are fully modular. However, by dropping the requirement of modularity, it is possible to define simpler versions of

\footnotetext{
${ }^{4}$ The definition can be extended to the propositional formulas of the language in the standard way [17].
} 


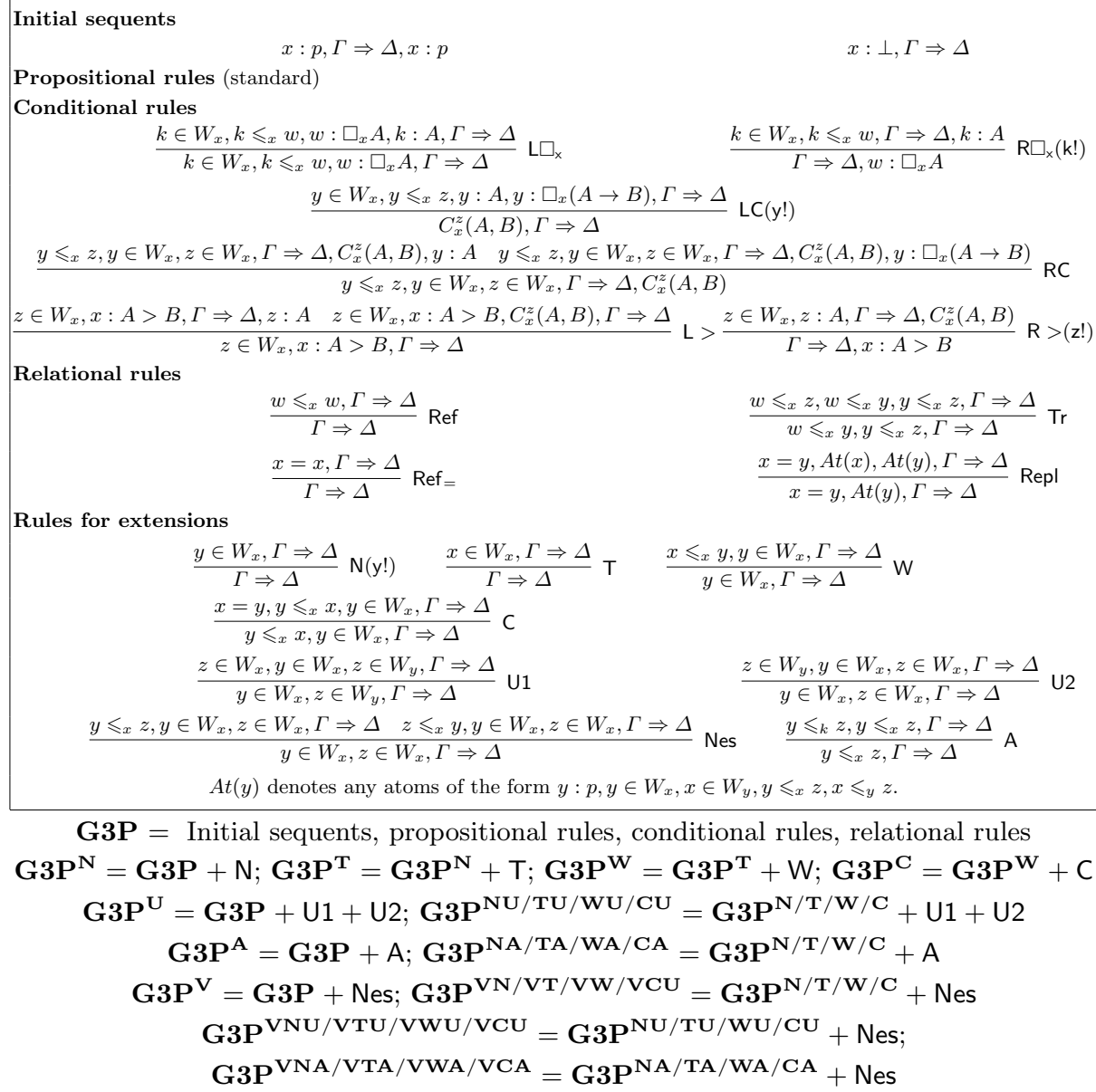

$\mathbf{G 3 P}=$ Initial sequents, propositional rules, conditional rules, relational rules

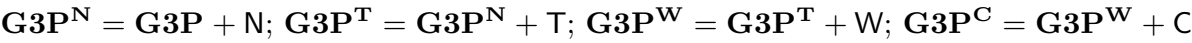

$\mathrm{G} \mathrm{P}^{\mathrm{U}}=\mathrm{G} 3 \mathrm{P}+\mathrm{U} 1+\mathrm{U} 2 ; \mathbf{G}^{\mathrm{N}} \mathbf{P}^{\mathrm{NU} / \mathrm{TU} / \mathrm{WU} / \mathrm{CU}}=\mathrm{G}^{\mathrm{N}} \mathbf{P}^{\mathrm{N} / \mathrm{T} / \mathrm{W} / \mathrm{C}}+\mathrm{U} 1+\mathrm{U} 2$

$\mathbf{G} 3 \mathbf{P}^{\mathbf{A}}=\mathbf{G} 3 \mathbf{P}+\mathrm{A} ; \mathbf{G} \mathbf{P}^{\mathbf{N A} / \mathbf{T A} / \mathrm{WA} / \mathbf{C A}}=\mathbf{G} \mathbf{3} \mathbf{P}^{\mathbf{N} / \mathbf{T} / \mathrm{W} / \mathbf{C}}+\mathrm{A}$

$\mathbf{G} 3 \mathbf{P}^{\mathbf{V}}=\mathbf{G} 3 \mathbf{P}+\mathrm{Nes} ; \mathbf{G} 3 \mathbf{P}^{\mathrm{VN} / \mathrm{VT} / \mathrm{VW} / \mathrm{VCU}}=\mathbf{G}^{\mathrm{N}} \mathbf{P}^{\mathrm{N} / \mathbf{T} / \mathrm{W} / \mathbf{C}}+\mathrm{Nes}$

$\mathrm{G3P}^{\mathrm{VNU} / \mathrm{VTU} / \mathrm{VWU} / \mathrm{VCU}}=\mathrm{G}^{\mathrm{V}} \mathbf{P}^{\mathrm{NU} / \mathrm{TU} / \mathrm{WU} / \mathrm{CU}}+\mathrm{Nes}$

$\mathrm{G3P}^{\mathrm{VNA} / \mathrm{VTA} / \mathrm{VWA} / \mathrm{VCA}}=\mathbf{G}^{\mathrm{N}} \mathbf{P}^{\mathrm{NA} / \mathrm{TA} / \mathrm{WA} / \mathrm{CA}}+\mathrm{Nes}$

Fig. 2. Rules of G3P*

the calculi for some subfamilies of the logics. In systems with uniformity it holds that for all $x \in W$ and $y \in W_{x}, W_{x}=W_{y}$. Thus, we can avoid specifying the relational atoms $y \in W_{x}$ in the rules (with this reformulation, the rules of uniformity would become superfluous). Similarly, in logics with absoluteness, uniformity holds, and moreover $w_{1} \leqslant_{x} w_{2}$ iff $w_{2} \leqslant y w_{1}$. Thus, we can avoid specifying the subscript $x$ in relational atoms $y \leqslant x z$, and the rule of absoluteness becomes superfluous. Finally, in the presence of nesting the truth condition for the conditional operator can be stated in a simpler way:

$x \Vdash A>B \equiv$ if there exists $z \in W_{x}$ such that $z \Vdash A$, then there exists $y \in W_{x}$ such that $y \Vdash A$ and $y \Vdash \square_{x}(A \rightarrow B)$.

Rules based on this truth condition, in addition to the simplification explained for uniformity, i.e., no relational atoms $y \in W_{x}$ and no rules $\mathrm{U} 1, \mathrm{U} 2$, yield the 
calculus proposed in [20], a proof system sound and complete with respect to the conditional logic $\mathbb{V} \mathbb{C U}$.

\section{Structural properties}

The height of a derivation is the number of nodes of the longest derivation branch, minus one. We recall that a rule is height-preserving admissible if whenever its premiss is derivable, the conclusion is also derivable with no greater derivation height. A rule is height-preserving invertible if whenever its conclusion is derivable, the premisses are derivable with no greater derivation height. Derivability with height bounded by $n$ is denoted by $\vdash_{n}$.

In order to prove admissibility of the structural rules we need a notion of label substitution given by, for instance, $x: A>B[y / x]=y: A>B$ and $w: \square_{x} A[y / x]=w: \square_{y} A$, extended component-wise to sequents, and a property of height-preserving substitution: If $\vdash_{n} \Gamma \Rightarrow \Delta$, then $\vdash_{n} \Gamma[y / x] \Rightarrow \Delta[y / x]$. Admissibility of generalized initial sequents (i.e., sequents of the form $x: A, \Gamma \Rightarrow$ $\Delta, x: A$ in which $A$ is not necessarily atomic) is shown by induction on the weight of $A$. We omit the routine proofs, the details of which are similar to those in [20].

The structural rules of weakening, contraction, and cut of $\mathbf{G 3} \mathbf{P}^{*}$ are the following:

$$
\begin{array}{cc}
\frac{\Gamma \Rightarrow \Delta}{\mathcal{F}, \Gamma \Rightarrow \Delta} \mathrm{Wk}_{\mathrm{L}} \frac{\Gamma \Rightarrow \Delta}{\Gamma \Rightarrow \Delta, \mathcal{F}} \mathrm{Wk}_{\mathrm{R}} \frac{\mathcal{F}, \mathcal{F}, \Gamma \Rightarrow \Delta}{\mathcal{F}, \Gamma \Rightarrow \Delta} \mathrm{Ctr}_{\mathrm{L}} \frac{\Gamma \Rightarrow \Delta, \mathcal{F}, \mathcal{F}}{\Gamma \Rightarrow \Delta, \mathcal{F}} \operatorname{Ctr}_{\mathrm{R}} \\
\frac{\Gamma \Rightarrow \Delta, \mathcal{F}}{\Gamma, \Gamma^{\prime} \Rightarrow \Delta^{\prime}} \\
\Gamma, \Gamma^{\prime} \Rightarrow \Delta, \Delta^{\prime}
\end{array}
$$

where $\mathcal{F}$ is a relational atom, a labelled formula, or a formula of the form $C_{x}^{z}(A, B)$. Observe that for $\mathrm{Wk}_{\mathrm{R}}, \mathrm{Ctr}_{\mathrm{R}}$, and cut we can without loss of generality omit the case of relational formulas since they never occur in the right-hand side of sequents. The calculi G3P* have the following structural properties:

\section{Theorem 2.}

i. All the rules are height-preserving invertible.

ii. The rules of weakening and contraction are height-preserving admissible.

iii. The rule of cut is admissible.

Proof.

$i$. By induction on the height of the derivation. Invertibility of relational rules, rules for extensions, $L \square_{x}, R C$ and $L>$ immediately follows from admissibility of weakening. Invertibility of the propositional rules and of $R \square_{x}$ is proved as in [17]; invertibility of LC is similar to that of the corresponding rule in [19].

ii. By induction on the height $n$ of the derivation. If $n=0$, the premiss of the contraction rule is an initial sequent, and so is its conclusion. If $n>0$, we look at the last rule $(R)$ applied. If $F$ is not principal in the rule, it suffices to apply the inductive hypothesis to the premiss of $(R)$, and then $(R)$. If $F$ is the 
principal formula of $R$, or was introduced by $R$, we distinguish two subcases. If $(R)$ is a rule in which the principal formula appears also in the premiss apply the hypothesis to the premiss, and then the rule. If $(R)$ is a rule in which the active formulas are subformulas of the principal formula, apply invertibility to the premiss(es) of the rule, then the inductive hypothesis, and $(R)$.

iii. By primary induction on the weight of the cut formula, and secondary induction on the sum of heights of the derivations of the premisses of cut. As usual, we proceed with a case distinction according to the last rule applied. If at least one of the premisses is an initial sequent, the conclusion of cut is also a sequent, or can be obtained by easy rule permutations. Similarly, if the cut formula is not principal in the last rule $R$ applied to one of the premiss of cut, the conclusion of cut can be obtained by permuting the cut upwards on the premiss of $R$, and then applying $R$ again. Finally, if the cut formula is principal in both rules applied to the premisses of cut, some more complex permutations are needed. Propositional cases can be found in [20]. We show only the case in which $R>$ and $L>$ are the rules applied to the left and right premiss of cut respectively. Consider a derivation ending with

$$
\frac{y \in W_{x}, y: A, \Gamma \Rightarrow \Delta, C_{x}^{y}(A, B)}{\frac{\Gamma \Rightarrow \Delta, x: A>B}{2} \mathrm{R}>\frac{z \in W_{x}, x: A>B, \Gamma^{\prime} \Rightarrow \Delta^{\prime}, z: A \quad z \in W_{x}, x: A>B, C_{x}^{y}(A, B), \Gamma^{\prime} \Rightarrow \Delta^{\prime}}{z \in W_{x}, x: A>B, \Gamma^{\prime} \Rightarrow \Delta^{\prime}} \mathrm{Lut}} \mathrm{L} \text { cu }
$$

Let $\mathcal{D}_{1}, \mathcal{D}_{2}, \mathcal{D}_{3}$ be the derivations ending with the topsequents above. The cut is transformed into four cuts of reduced rank as follows. First we have two cuts, to topmost of reduced height, the second of reduced weight, where $\mathcal{D}_{1}[z / y]$ denotes the derivation resulting from $\mathcal{D}_{1}$ by application of an height-preserving substitution:

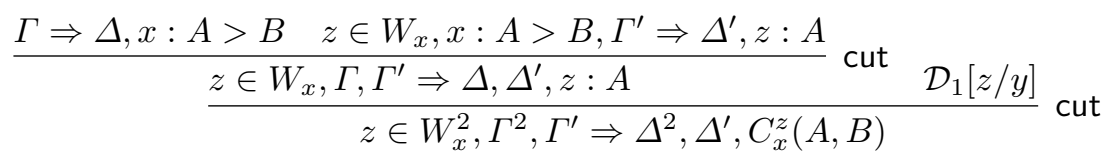

Second we have the cut of reduced height

$$
\frac{\Gamma \Rightarrow \Delta, x: A>B \quad \mathcal{D}_{3}}{z \in W_{x}, \Gamma, \Gamma^{\prime}, C_{x}^{z}(A, B) \Rightarrow \Delta, \Delta^{\prime}} \text { cut }
$$

Finally, by cut their conclusions through a fourth cut of reduced weight and obtain the sequent $z \in W_{x}^{3}, \Gamma^{3}, \Gamma^{\prime 2} \Rightarrow \Delta^{3}, \Delta^{\prime 2}$. Admissible weakening steps give the conclusion of the original cut.

The case of principal cut formula of the form $C_{x}^{z}(A, B)$ is reduced in a similar way through four cuts, the uppermost of reduces height, and the lowermost of reduces weight. For principal formula of the form of an indexed modality, the conversion is the standard one for the necessity modality of labelled calculi.

Thanks to admissibility of cut, it is possible to prove the following:

Theorem 3 (Completeness). If a formula $A$ is valid in preferential models and extensions, then sequent $\Rightarrow x: A$, for an arbitrary label $x$, is derivable in the corresponding $\mathbf{G} 3 \mathbf{P}^{*}$ calculus. 
Proof. By using the known completeness result for extensions of $\mathbb{P} \mathbb{C}$ w.r.t. preferential models and showing that the inference rules of $\mathbb{P C L}$ are admissible in G3P, and that the axioms of $\mathbb{P C L}$ and its extensions are derivable in the corresponding proof system of $\mathbf{G} 3 \mathbf{P}^{*}$. The proof for $\mathbb{P C L}$ is similar to the proof in [20]. By way of example, we show the derivation of Axiom $\mathrm{U}_{1}$ in $\mathbf{G} \mathbf{3} \mathbf{P} \mathbf{V}$, omitting the derivable left premiss of $L>$.

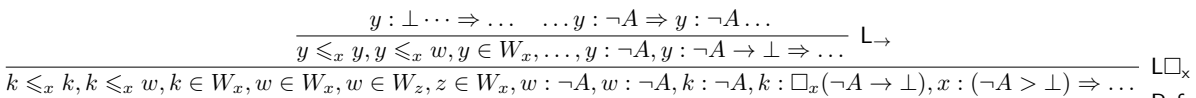

$$
\begin{aligned}
& \frac{k \leqslant_{x} w, k \in W_{x}, w \in W_{x}, w \in W_{z}, z \in W_{x}, w: \neg A, w: \neg A, k: \neg A, k: \square_{x}(\neg A \rightarrow \perp), x:(\neg A>\perp) \Rightarrow \ldots}{w \in W_{x}, w \in W_{z}, z \in W_{x}, w: \neg A, w: \neg A, C^{w}(\neg A, \perp), x:(\neg A>\perp) \Rightarrow \ldots} \mathrm{LC} \\
& \frac{w \in W_{x}, w \in W_{z}, z \in W_{x}, w: \neg A, w: \neg A, C_{x}^{w}(\neg A, \perp), x:(\neg A>\perp) \Rightarrow \ldots}{w \in W_{x}, w \in W_{z}, z \in W_{x}, w: \neg A, x:(\neg A>\perp) \Rightarrow \ldots} \mathrm{L}> \\
& \overline{w \in W_{z}, z \in W_{x}, w: \neg A, x:(\neg A>\perp) \Rightarrow C_{x}^{z}(\neg(\neg A>\perp), \perp), C_{z}^{w}(\neg A, \perp)} \mathrm{U} 1 \\
& \frac{z \in W_{x}, x:(\neg A>\perp) \Rightarrow z: \neg A>\perp, C_{x}^{z}(\neg(\neg A>\perp), \perp)}{z \in W_{x}, z: \neg(\neg A>\perp), x:(\neg A>\perp) \Rightarrow C_{x}^{z}(\neg(\neg A>\perp), \perp)} \mathrm{R} \neg \\
& \frac{z \in W_{x}, z: \neg(\neg A>\perp), x:(\neg A>\perp) \Rightarrow C_{x}^{2}(\neg(\neg A>\perp), \perp)}{x:(\neg A>\perp) \Rightarrow x: \neg(\neg A>\perp)>\perp} \mathrm{R}> \\
& \frac{x:(\neg A>\perp) \Rightarrow x: \neg(\neg A>\perp)>\perp}{\Rightarrow x:(\neg A>\perp) \rightarrow \neg(\neg A>\perp)>\perp} \mathrm{R}_{\rightarrow}
\end{aligned}
$$

By Theorem 1, Theorem 3, and the known completeness results for $\mathbb{P} \mathbb{C}$ and its extensions with respect to preferential models, we have:

Corollary 1. Formula $A$ is provable in any of the systems of the conditional logics cube if and only if $\Rightarrow x: A$ is derivable in the corresponding labelled system.

\section{Termination and completeness}

In this section, we shall give an alternative direct proof of completeness for the calculi G3P, G3P $\mathbf{P}^{\mathrm{N}}, \mathrm{G} 3 \mathrm{P}^{\mathrm{T}}, \mathrm{G} 3 \mathbf{P}^{\mathrm{W}}, \mathrm{G}^{\mathrm{C}} \mathbf{P}^{\mathrm{C}}$ and G3PV, G3PVN, G3PVT, G3PVW $\mathbf{P}^{\mathbf{V W}} \mathbf{G P}^{\mathbf{V C}}$ (from now on $\mathbf{G} 3 \mathbf{P}^{\mathbf{V} / \mathbf{N} / \mathbf{T} / \mathbf{W} / \mathbf{C}}$ ), i.e., the systems without uniformity and absoluteness. ${ }^{5}$ The proof proceeds by showing how to construct a countermodel from failed proof search. We first need to prove that root-first proof search, which in general is not terminating because of loops, terminates under the adoption of a suitable strategy.

Example 1. Loop generated by repeated applications of rule $\mathrm{L}>$ and $\mathrm{LC}$ (only the right premisses of $\mathrm{L}>$ are shown).

$$
\frac{y \leqslant x z, y \in W_{x}, z \leqslant{ }_{x} z, z \in W_{x}, y: A, y: \square_{x}(A \rightarrow B), x: A>B, x: C>D, C_{x}^{z}(C, D), C_{x}^{y}(C, D) \Rightarrow}{\frac{y \leqslant x}{z, y \in W_{x}, z \leqslant{ }_{x} z, z \in W_{x}, y: A, y: \square_{x}(A \rightarrow B), x: A>B, x: C>D, C_{x}^{z}(C, D) \Rightarrow} \mathrm{L}} \mathrm{L}>
$$

\footnotetext{
5 The proofs of termination and completeness for systems with Uniformity and Absoluteness can be given adopting the reformulation of the calculi from Remark 1 . The proofs for the current versions of the calculi would be unnecessarily complex.
} 
To ensure termination we introduce the notion of saturated sequent, a sequent to which all the rules have been applied in a non-redundant way. We then specify a proof search strategy, blocking the application of the rules to a saturated sequent.

Definition 5. Given a $\mathbf{G} 3 \mathbf{P}^{\mathbf{V} / \mathbf{N} / \mathbf{T} / \mathbf{W} / \mathbf{C}}$ derivation, let $\mathcal{B}=S_{0}, S_{1}, \ldots$ be a derivation branch, with $S_{i}$ sequent $\Gamma_{k} \Rightarrow \Delta_{k}$ for $k>0$, and $S_{0}$ sequent $\Rightarrow x_{0}: A_{0}$. Let $\downarrow \Gamma_{k} / \downarrow \Delta_{k}$ be the union of the antecedents / succedents occurring in the derivation from $S_{0}$ up to $S_{k}$. A sequent $\Gamma \Rightarrow \Delta$ is saturated if it is not an instance of an initial sequent, and the following conditions are satisfied:

$\left(\mathrm{L}_{\rightarrow}\right)$ If $x: A \rightarrow B$ occurs in $\downarrow \Gamma, x: B$ occurs in $\downarrow \Gamma$ or $x: A$ occurs in $\downarrow \Delta$;

$\left(\mathrm{R}_{\rightarrow}\right)$ If $x: A \rightarrow B$ occurs in $\downarrow \Delta, x: A$ occurs in $\downarrow \Gamma$ and $x: B$ occurs in $\downarrow \Delta ;^{6}$

(Ref) If $y$ occurs in $\Gamma$, then $y \leqslant_{x} y$ occurs in $\Gamma$;

(Tr) If $y \leqslant_{x} z$ and $z \leqslant{ }_{x} k$ occur in $\Gamma, y \leqslant{ }_{x} k$ occur in $\Gamma$;

$(\mathrm{L}>)$ If $x: A>B$ and $z \in W_{x}$ occur in $\downarrow \Gamma$, then either $z: A$ occurs in $\downarrow \Delta$ or $C_{x}^{z}(A, B)$ occurs in $\downarrow \Gamma$;

$(\mathrm{R}>)$ If $x: A>B$ occurs in $\downarrow \Delta$, then $z \in W_{x}$ and $z: A$ occur in $\downarrow \Gamma$, for some $z$;

(LC) if $C_{x}^{z}(A, B)$ occurs in $\Gamma$, then either for some $y y \leqslant x z, y \in W_{x}, y$ : $\square_{x}(A \rightarrow B)$ occur in $\downarrow \Gamma$, or for some $w$ such that $z \neq w, z \leqslant x$ and $C_{x}^{w}(A, B)$ occur in $\downarrow \Gamma$;

(RC) If $y \leqslant x z, y \in W_{x}, z \in W_{x}$ occur in $\downarrow \Gamma$ and $C_{x}^{z}(A, B)$ occurs in $\downarrow \Delta$, then either $y: A$ or $y: \square_{x}(A)$ occurs in $\downarrow \Delta$;

$\left(\mathrm{L} \square_{\times}\right)$If $y: \square_{x} A$ occurs in $\downarrow \Gamma, z \leqslant x y$ and $z \in W_{x}$ occur in $\Gamma$, and $z: A$ occurs in $\downarrow \Gamma$;

$\left(\mathrm{R} \square_{\mathrm{x}}\right)$ If $y: \square_{x} A$ occurs in $\downarrow \Delta$, then either for some $z, z \leqslant x$ occurs in $\Gamma$ and $z: A$ occurs in $\downarrow \Delta$, or for some $w \neq y, y \leqslant x$ occurs in $\Gamma$ and $w: \square_{x} A$ occurs in $\downarrow \Delta$;

(N) If $x$ occurs in $\Gamma, y \in W_{x}$ occurs in $\Gamma$, for some $y$;

(T) If $x$ occurs in $\Gamma, x \in W_{x}$ occurs in $\Gamma$;

(W) If $y \in W_{x}$ occurs in $\Gamma, x \leqslant x y$ occurs in $\Gamma$;

(C) If $y \leqslant_{x} x$ and $y \in W_{x}$ occur in $\Gamma, y=x$ occurs in $\Gamma$;

$\left(\operatorname{Ref}_{=}\right)$If $x$ occurs in $\Gamma$, then $x=x$ occurs in $\Gamma$;

(Repl) If $y=x$ occurs in $\Gamma$, and if some formulas At( $y)$ occur in $\Gamma$, formulas At $(x)$ occur in $\Gamma$;

(Nes) If $y \in W_{x}$ and $z \in W_{x}$ occur in $\Gamma, y \leqslant_{x} z$ or $z \leqslant x$ occur in $\Gamma$.

In Example 1, the saturation condition (LC) blocks the application of the rule to formula $C_{x}^{y}(C, D)$, since $y \leqslant_{x} z$ and $C_{x}^{z}(C, D)$ occur in the antecedent. Intuitively, rule LC is not applied to a formula $C_{x}^{y}(C, D)$ if $y$ has been generated by a previous application of LC to the same $C_{x}(C, D)$, possibly labelled with a different label, i.e., if for some $z$, formulas $y \leqslant_{x} z$ and $C_{x}^{z}(C, D)$ occur in a lower antecedents. A similar saturation condition is needed for $\mathrm{R} \square_{\mathrm{x}}$.

Definition 6. In root-first proof search for $\Rightarrow x_{0}: A_{0}$, apply the following:

\footnotetext{
${ }^{6}$ The saturation conditions for the other propositional rules are standard [20].
} 
1. Rules which do not introduce new labels are applied before rules which do introduce new labels;

2. A rule $R$ cannot be applied to a sequent if the sequent already satisfies the saturation condition associated to $R$.

We need to show that every branch of a derivation starting with $\Rightarrow x_{0}: A_{0}$ and built in accordance with the strategy is finite. Since labels can be attached only to the finitely many subformulas of formula $A_{0}$, it suffices to prove that only a finite number of labels can occur in the branch. To this aim, we construct an acyclic graph with the labels occurring in the derivation, and show that the graph is finite: more precisely, that every node of the graph has a finite number of immediate successors, and that each branch of the graph is finite.

Definition 7. Given a derivation branch as in Definition 5, let $x, y$ be labels occurring in $\Gamma$. Let $k(x)=\min \left\{t \mid x\right.$ occurs in $\left.\Gamma_{t}\right\}$. We say that " $x$ generates $y$ ", in symbols $x R y$, if for some $t \geqslant k(x), k(y)=t$ and $y \in W_{x}$ occurs in $\Gamma_{t}$.

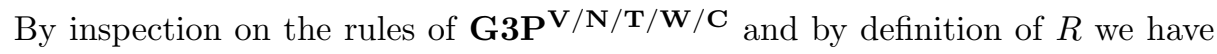
that the relation $R$ does not contain any cycles and forms a graph having at the root label $x_{0}$, and that all the labels occurring in the derivation occur in the graph. The notion of conditional degree, needed to prove Lemma 2, corresponds to the level of nesting of the conditional operator $>$.

Definition 8. The conditional degree of a formula $A$ is defined as: $d(\perp)=$ $d(p)=0 ; d(A \circ B)=d\left(C_{x}^{y}(A, B)\right)=\max (d(A), d(B))$ for $\circ=\{\wedge, \vee, \rightarrow\} ;$ $d\left(\square_{k} A\right)=d(A)$, and $d(A>B)=\max (d(A), d(B))+1$. For $x$ a label in $a$ derivation, $d(x)=\max \{d(C) \mid x: C$ occurs in $\downarrow \Gamma \cup \downarrow \Delta\}$.

Lemma 1. Every node in the graph generated by the relation $R$ has a finite number of immediate successors.

Proof. By definition, label $y$ is generated from $x$ if there exists a $t$ such that $y$ does not occur in $\Gamma_{s}$ for any $s<t$, and $y \in W_{x}$ occurs in $\Gamma_{t}$. We need to prove that only a finite number of formulas $y \in W_{x}$ can be introduced from $x$.

Formulas $y \in W_{x}$ are introduced in root-first proof search by application of rules for semantic conditions, $\mathrm{R}>$, $\mathrm{LC}$ or $\mathrm{R} \square_{\mathrm{x}}$. In the first cases, $y \in W_{x}$ is introduced by $\mathrm{N}$ or $\mathrm{T} .{ }^{7}$ By the saturation conditions, these rules can be applied at most once to a label $x$; thus, they generate at most 2 new labels. If $y \in W_{x}$ is introduced by $\mathrm{R}>$, the rule must have been applied to some $x: C>D$ occurring in $\Delta_{t-1}$. By the saturation condition, rule $\mathrm{R}>$ can be applied at most once to each formula $x: C>D$, and the number of such formulas linearly depends on $d\left(A_{0}\right)$, the degree of formula $A_{0}$ at the root of the tree. Similarly, rule $\mathrm{R} \square_{\mathrm{x}}$ is applied to some $w: \square_{A}$ in $\downarrow \Gamma_{t-1}$, generating a new $y \in W_{x}$. Formulas $w: \square_{A}$ are introduced by $\mathrm{RC}$ and $\mathrm{R}>$, which do not generate loops. The saturation

\footnotetext{
7 Observe that Repl does not introduce new labels; however, it could introduce new link between the nodes of the graph. In the presence of Repl the structure generated by $R$ is a graph; otherwise, it is a tree.
} 
condition $\left(R \square_{x}\right)$ ensures that no loops arise with formulas $L \square_{x}$. Thus, only a finite number of labels can be introduced. In case $y \in W_{x}$ is introduced by LC the situation is more complex. As shown in Example 1, rule LC might interact with rule $L>$ generating a large number of new labels, however, thanks to the proof search strategy, their number is finite. We consider a case of loop more complex than the one in Example 1: suppose formulas $x: E_{1}>F_{1}, \ldots, x: E_{k}>F_{k}$ occur in the succedent of a sequent. Then, for some $z \in W_{x}$ in the antecedent, we can apply $k$ times rule $\mathrm{L}>$, generating $k$ formulas $C_{x}^{z}\left(E_{1}, F_{1}\right), \ldots, C_{x}^{z}\left(E_{k}, F_{k}\right)$. Then, rule LC can be applied to these formulas, generating $k$ new labels $z_{1} \in$ $W_{x}, \ldots, z_{k} \in W_{x}$, with $z_{1} \leqslant z, \ldots, z_{k} \leqslant x$. Moreover, the rule introduces in the antecedent formulas $z_{1}: E_{1}, \ldots, z_{k}: E_{k}$ and $z_{1}: \square_{x}\left(E_{1} \rightarrow F_{1}\right), \ldots, z_{k}$ : $\square_{x}\left(E_{k} \rightarrow F_{k}\right)$. Rule $\mathbf{L}>$ can be applied to these labels, generating $k \cdot k$ new formulas:

$$
\begin{gathered}
C_{x}^{z_{1}}\left(E_{1}, F_{1}\right), \ldots, C_{x}^{z_{1}}\left(E_{k}, F_{k}\right) \\
\vdots \\
C_{x}^{z_{k}}\left(E_{1}, F_{1}\right), \ldots, C_{x}^{z_{k}}\left(E_{k}, F_{k}\right)
\end{gathered}
$$

Application of $L C$ to these formulas would in principle generate $k \cdot k$ new labels; however, the saturation condition (LC) blocks the application of the rule to all formulas. For $1 \leqslant i \leqslant k$ and $1 \leqslant j \leqslant k$, consider formula $C_{x}^{z_{i}}\left(E_{j}, F_{j}\right)$. It holds that formulas $z_{i} \leqslant x z$ and $C_{x}^{z}\left(E_{j}, F_{j}\right)$ occur in lower antecedents/succedents, satisfying the saturation condition. Thus, for each $y \in W_{x}$, and for $k>$-formulas occurring in the antecedent $k \cdot k$ new labels are generated.

Lemma 2. Every branch in the graph generated by the relation $R$ is finite.

Proof. By induction on $d(x)$, for $x$ a label in the graph. We show that the length of an arbitrary chain starting from $x$ is bounded by the degree of the formula it labels. If $d(x)=0$, the formulas labelled with $x$ are all atomic or propositional formulas, and no formula $y \in W_{x}$ needs to be introduced. If $d(x)>0$, there must be some formula $x: A>B$ occurring in $\downarrow \Gamma \cup \downarrow \Delta$. Thus, there is at least one chain of length greater than zero in the branch, and some label $y$ such that $x R y$. Observe that $y$ can occur only as label of formulas of smaller degree than the formulas labelled with $x$. More precisely, for all formulas $x: A>B$ with $d(A>B) \leqslant d(x)$ occurring in $\downarrow \Gamma \cup \downarrow \Delta$, it holds that for all formulas $y: \square_{x}(A \rightarrow B), d(y)<d(x)$, i.e., all labels introduced by combination of $\mathrm{R}>$, $\mathrm{L}>$, LC, RC and $\mathrm{R} \square_{\mathrm{x}}$ are label of formulas with a smaller degree than formulas labelled with $x$.

It follows from Lemmas 1 and 2 that the acyclic graph is finite. Since the formulas occurring in a derivation are subformulas of the formula $A_{0}$, and since the number of labels occurring in a derivation is finite, proof search terminates.

Theorem 4 (Termination). Proof search in $\mathbf{G} 3 \mathbf{P}^{\mathbf{V} / \mathbf{N} / \mathbf{T} / \mathbf{W} / \mathbf{C}}$ built in accordance with the proof search strategy for a sequent $\Rightarrow x_{0}: A_{0}$ always comes to an end in a finite number of steps, and each sequent occurring as a leaf of the derivation tree is either an initial sequent or a saturated sequent. 
Termination of proof search allows to prove completeness by constructing a countermodel from a saturated sequent.

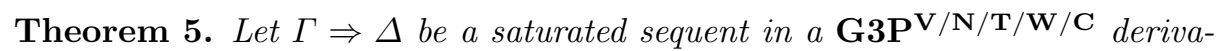
tion. There exists a finite countermodel $\mathcal{M}_{\mathcal{B}}$ satisfying all formulas in $\downarrow \Gamma$ and falsifying all formulas in $\downarrow \Delta$.

Proof. The countermodel $\mathcal{M}_{\mathcal{B}}$ is constructed as follows: $W_{\mathcal{B}}=\{x \mid x$ occurs in $\downarrow$ $\Gamma \cup \downarrow \Delta\}$; for all $x \in W_{\mathcal{B}}, W_{x}=\left\{y \mid y \in W_{x}\right.$ occurs in $\left.\Gamma\right\} ; \leqslant x=\{\langle y, z\rangle \mid y \leqslant x$ $z$ occurs in $\Gamma\}$; for $p$ atomic, $\llbracket p \rrbracket=\left\{x \in W_{\mathcal{B}} \mid x: p\right.$ occurs in $\left.\Gamma\right\}$.

It is immediate to verify that the relation $\leqslant_{x}$ satisfies the properties of reflexivity and transitivity; thus, $\mathcal{M}_{\mathcal{B}}$ is a model for $\mathbb{P C L}$. In the presence of $\mathrm{N}$, $\mathrm{T}, \mathrm{W}, \mathrm{C}$ and Nes, the saturation conditions associated to these rules ensure that the model $\mathcal{M}_{\mathcal{B}}$ is a model for the corresponding logic. ${ }^{8}$

Let $\rho$ be the realization $\rho(x)=x$. We show that 1) if $\mathcal{F}$ occurs in $\downarrow \Gamma$, $\mathcal{M}_{\mathcal{B}} \vDash_{\rho} \mathcal{F}$, and 2) if $\mathcal{F}$ occurs in $\downarrow \Delta, \mathcal{M}_{\mathcal{B}} \not \nvdash_{\rho} \mathcal{F} .{ }^{9}$. The two claims are proved by cases, and by induction on the weight of $\mathcal{F}$. If $\mathcal{F}$ is a relational atom $y \in W_{x}$ or $y \leqslant x z$ or a formula $x: p$, claim 1 (and claim 2) hold by definition of the model. The propositional cases and the cases of $\mathcal{F}=x: A>B$ and $\mathcal{F}=y: \square_{x} A$ follow applying the inductive hypothesis. By way of example, we prove claim 2 for $\mathcal{F}=x: A>B$. Suppose that formula $x: A>B$ occurs in $\downarrow \Delta$. By the saturation condition associated to $\mathrm{R}>{ }_{1}$, for some label $z, z \in W_{x}$ and $z: A$ occur in $\downarrow \Gamma$. Thus, by inductive hypothesis, $\mathcal{M}_{\mathcal{B}} \vDash_{\rho} z: A$. Moreover, by the saturation conditions associated to Ref and $\mathrm{R}>_{2}$, either $z: A$ occurs in $\downarrow \Delta$, or $z: \square_{x}(A \rightarrow B)$ occurs in $\downarrow \Delta$. By inductive hypothesis, either $\mathcal{M}_{\mathcal{B}} \not \nvdash_{\rho} z: A$ or $\mathcal{M}_{\mathcal{B}} \not \not_{\rho} z: \square_{x}(A \rightarrow B)$. Thus, by definition $\mathcal{M}_{\mathcal{B}} \not \not_{\rho} x: A>B$.

As a consequence of Theorem 4 and 5 we have that any underivable sequent originates, in a finite number of steps, a saturated sequent which is used to define a countermodel. We therefore have:

Corollary 2 (Strong completeness). Any sequent $\Gamma \Rightarrow \Delta$ is either derivable in $\mathbf{G} 3 \mathbf{P}^{\mathbf{V}} / \mathbf{N} / \mathbf{T} / \mathbf{W} / \mathbf{C}$ or has a (finite) countermodel in the corresponding class of models.

Completeness of the proof systems is an obvious consequence:

Theorem 6 (Completeness). If $A$ is valid in one of the logics without uniformity and absoluteness, sequent $\Rightarrow x: A$ is derivable in the corresponding $\mathbf{G} 3 \mathbf{P}^{\mathbf{V} / \mathbf{N} / \mathbf{T} / \mathbf{W} / \mathbf{C}}$ calculus.

${ }^{8}$ In case of centering it is convenient to define worlds as equivalence classes, to account for formulas $x=y$. Thus, $[x]=\{y \mid x=y$ occurs in $\downarrow \Gamma\}$ and $W^{c}=\{[x] \mid$ $y$ occurs in $\downarrow \Gamma \cup \downarrow \Delta\}$. Centering follows from the saturation condition (C).

${ }^{9}$ In case of centering, we also need to show that if $[x] \vDash_{\rho} A$ and $y \in[x]$, then $[y] \vDash_{\rho} A$, and that if $[x] \vDash_{\rho} A$ then $x: A$ occurs in $\downarrow \Gamma$. The proof follows from admissibility of Repl in its generalized form [20] 
Completeness, along with termination, allow to define a decision procedure for the logics based on the labelled calculi. However, the resulting decision procedure would be of at least NEXPTIME complexity - thus, far from the known complexity bounds for the logics. ${ }^{10}$

\section{Conclusion and related work}

In this work, we introduced a family of uniform labelled calculi that capture in a modular way the conditional logic $\mathbb{P} \mathbb{L}$ and its extensions, including Lewis' counterfactual systems. The calculi internalise the semantics of preferential models. This semantics, studied, among other, by Lewis and Burgess [15,5], makes explicit reference to the comparative plausibility ordering among worlds, implicitly assumed in Lewis's sphere models.

Several labelled proof system for conditional logics have been defined in the literature. A recent approach, based on the methodology of neighbourhood semantics of [18] and [19], is presented in [10] and gives a uniform family of labelled calculi for $\mathbb{P} \mathbb{C L}$ and its extensions. Neighbourhood semantics is a generalization of Lewis's sphere semantics; whereas the latter is adequate for $\mathbb{V}$, neighbourhood semantics covers also weaker conditional logics. When compared to labelled calculi based on neighbourhood semantics, the calculi G3P* appear to be simpler: they use just one set of labels, whereas the calculi based on neighbourhood semantics need two sets of labels, for worlds and for neighbourhoods. It turns out that the preferential semantics already used to define labelled sequent calculi for Lewis's conditional logic $\mathbb{V} \mathbb{C}$ in [20], is sufficiently expressive to treat uniformly also the weaker extensions of $\mathbb{P C L}$.

Preferential models have already been used in [9] to define tableau calculi for $\mathbb{P C L}$ and all its extensions, but with the important difference, with respect to our approach, of the addition of the Limit Assumption ${ }^{11}$ and the use of a strict relation of comparative similarity. Another semantically inspired approach can be found in [21], presenting a sequent calculus for system $\mathbb{C K}$ and some of its extensions. These logics are the weakest conditional systems, and they are weaker than the logics considered in this article.

Internal calculi (i.e., proof systems in which sequents have a direct formula interpretation) for conditional logics have also been defined: in [2] nested sequent calculi for $\mathbb{C K}$ and some of its extensions are devloped, whereas in [1] a nested and optimal calculus for counterfactual logic $\mathbb{V}$ can be found (refer to [11] for cases of extensions). Finally, display calculi for $\mathbb{C} \mathbb{K}$ have been introduced recently in $[6]$.

With respect to the labelled proof systems $\mathbf{G 3 P}^{*}$, the internal calculi are less modular: they capture weaker logics, such as $\mathbb{C K}$, or subfamilies of the logics considered in this article, such as $\mathbb{V}$ and its extensions. In particular, the definition of internal calculi for $\mathbb{P C L}$ seems challenging: up to now, the only internal proof system known for it is the resolution calculus presented in [16].

$\overline{10}$ Refer to [7] for complexity results for conditional logics.

11 The Limit Assumption states that there are no infinite descending $\leqslant x_{x}$-chains. 
The labelled approach treats in a modular way both $\mathbb{P C L}$ and $\mathbb{V}$. The challenge of defining labelled calculi on the basis of preferential semantics lies in identifying a decomposition of the conditional operator in terms of simpler operators directly treatable by the sequent calculus rules. Here, this is done by introducing the indexed operator $\square_{x} A$, similarly to $[9,20]$, and the binary operator $C_{x}^{z}(A, B)$.

As underlined in Remark 1, by dropping the requirement of modularity it is possible to have simpler labelled calculi for sub-families of logics. We plan to define such calculi and analyse their termination in root-first proof search, to investigate the possibility of a better complexity bound for the corresponding logics. Furthermore, following [9], simpler rules could be defined also for G3P with the introduction of Limit Assumption on preferential models. Finally, we plan to study the relationship of $\mathbf{G 3 P}^{*}$ with labelled sequent calculi for conditional logics based on neighbourhood models [10]. Via the correspondence between neighbourhood and preferential structures [3], we conjecture that the two families of calculi can be proved equivalent. It would be interesting to know which family of calculi allows for the optimal decision procedure.

\section{References}

1. Alenda, R., Olivetti, N., Pozzato, G.L.: Nested sequent calculi for conditional logics. In: European Workshop on Logics in Artificial Intelligence. pp. 14-27. Springer (2012)

2. Alenda, R., Olivetti, N., Pozzato, G.L.: Nested sequent calculi for normal conditional logics. Journal of Logic and Computation 26(1), 7-50 (2013)

3. Alexandroff, P.: Diskrete Räume. Mat.Sb. (NS) 2(3), 501-519 (1937)

4. Baltag, A., Smets, S.: A qualitative theory of dynamic interactive belief revision. Logic and the foundations of game and decision theory (LOFT 7) 3, 9-58 (2008)

5. Burgess, J.P.: Quick completeness proofs for some logics of conditionals. Notre Dame Journal of Formal Logic 22(1), 76-84 (1981)

6. Chen, J., Greco, G., Palmigiano, A., Tzimoulis, A.: Non normal logics: semantic analysis and proof theory. arXiv preprint arXiv:1903.04868 (2019)

7. Friedman, N., Halpern, J.Y.: On the complexity of conditional logics. In: Doyle, J., Sandewall, E., Torasso, P. (eds.) Principles of knowledge Representation and Reasoning: Proceedings of the Fourth International Conference (KR'94). pp. 202 213. Morgan Kaufmann Pub (1994)

8. Galles, D., Pearl, J.: An axiomatic characterization of causal counterfactuals. Foundations of Science 3(1), 151-182 (1998)

9. Giordano, L., Gliozzi, V., Olivetti, N., Schwind, C.: Tableau calculus for preferencebased conditional logics: PCL and its extensions. ACM Transactions on Computational Logic 10(3), 21 (2009)

10. Girlando, M.: On the proof theory of conditional logics. Ph.D. thesis, Univeristy of Helsinki (2019)

11. Girlando, M., Lellmann, B., Olivetti, N., Pozzato, G.L.: Standard sequent calculi for Lewis' logics of counterfactuals. In: European Conference on Logics in Artificial Intelligence. pp. 272-287. Springer (2016)

12. Girlando, M., Negri, S., Olivetti, N., Risch, V.: Conditional beliefs: from neighbourhood semantics to sequent calculus. The Review of Symbolic Logic pp. 1-44 (2018) 
13. Grahne, G.: Updates and counterfactuals. Journal of Logic and Computation 8(1), 87-117 (1998)

14. Kraus, S., Lehmann, D., Magidor, M.: Nonmonotonic reasoning, preferential models and cumulative logics. Artificial intelligence 44(1-2), 167-207 (1990)

15. Lewis, D.K.: Counterfactuals. Blackwell, Oxford (1973)

16. Nalon, C., Pattinson, D.: A resolution-based calculus for preferential logics. In: International Joint Conference on Automated Reasoning. pp. 498-515. Springer (2018)

17. Negri, S.: Proof analysis in modal logic. Journal of Philosophical Logic 34(5-6), $507(2005)$

18. Negri, S.: Proof theory for non-normal modal logics: The neighbourhood formalism and basic results. IFCoLog Journal of Logics and their Applications 4, 1241-1286 (2017)

19. Negri, S., Olivetti, N.: A sequent calculus for preferential conditional logic based on neighbourhood semantics. In: International Conference on Automated Reasoning with Analytic Tableaux and Related Methods. pp. 115-134. Springer (2015)

20. Negri, S., Sbardolini, G.: Proof analysis for Lewis counterfactuals. The Review of Symbolic Logic 9(1), 44-75 (2016)

21. Poggiolesi, F.: Natural deduction calculi and sequent calculi for counterfactual logics. Studia Logica 104(5), 1003-1036 (2016)

22. Weiss, Y.: Frontiers of Conditional Logic. Ph.D. thesis, The Graduate Center, City University of New York (2019) 